\title{
BMJ Open Quality Hospital accreditation processes in Saudi Arabia: a thematic analysis of hospital staff experiences
}

\author{
Ali Al Mansour, ${ }^{1}$ Alan F Merry, ${ }^{2,3}$ Tanisha Jowsey, ${ }^{4}$ Jennifer M Weller ${ }^{3,4}$
}

To cite: Al Mansour A, Merry AF, Jowsey T, et al. Hospital accreditation processes in Saudi Arabia: a thematic analysis of hospital staff experiences. BMJ Open Quality 2022;11:e001652. doi:10.1136/ bmjoq-2021-001652

Received 24 August 2021 Accepted 20 December 2021

Check for updates

(C) Author(s) (or their employer(s)) 2022. Re-use permitted under CC BY-NC. No commercial re-use. See rights and permissions. Published by BMJ.

${ }^{1}$ Quality Management, Saudi Arabia Ministry of Health,

Riyadh, Saudi Arabia

${ }^{2}$ Department of Anaesthesiology, The University of Auckland School of Medicine, Auckland, Auckland, New Zealand ${ }^{3}$ Department of Anaesthesia, Auckland City Hospital, Auckland, New Zealand ${ }^{4}$ Centre for Medical and Health Sciences Education, The University of Auckland School of Medicine, Auckland, New Zealand

Correspondence to

Dr Ali Al Mansour;

almansour.ali@hotmail.com

\section{ABSTRACT}

Background Hospital accreditation by an international organisation can play an important role in health quality and safety. However, little is known about how managers and front-line employees experience and perceive the effects of accreditation. Their views could inform quality improvement processes and procedures.

Objective To explore perceptions of employees at the managerial level on the Joint Commission International (JCl) accreditation process and its impact on quality of patient care in Saudi Arabian JCl-accredited hospitals. Methods We undertook a qualitative study using semi-structured interviews to explore the perspectives of senior staff from three accredited public hospitals in Saudi Arabia. Interviews were transcribed prior to thematic analysis.

Results Twenty managers participated in the interviews. The following inter-related themes emerged concerning the $\mathrm{JCl}$ accreditation process and its impact on quality of patient care: drivers for the change; the plan for the change; the process of the change; maintaining changes post-accreditation and patients' issues. Participants were positive in their accounts of: drivers for the change; planning for the change needed to achieve accreditation and managing patients' issues. However, participants reported less favourably on: the process of the change; and maintaining changes postaccreditation.

Conclusion The planning stage was perceived as the easiest component of $\mathrm{JCl}$ accreditation. Implementing and maintaining changes post-accreditation that demonstrably promote patient safety and quality of care was perceived as more difficult. When planning for accreditation, institutions need to incorporate strategies to ensure that improvements to care continue beyond the accreditation period.

\section{INTRODUCTION}

The intention of hospital accreditation is to ensure that hospitals meet international standards of quality and safety in patient care. There is some evidence that the accreditation process can improve quality and safety. ${ }^{1-5}$ These studies have employed a range of methods to collect this evidence, including: surveys of patient satisfaction, staff perception, managers' surveys and clinical quality measures. However, there is very limited evidence from qualitative methods, such as interviews with managers or frontline employees, to explore their experiences of hospital accreditation by an international organisation or their views on the impact on quality of patient care ${ }^{67}$ Interviews have the potential to uncover and generate a detailed understanding of respondents' experiences of the process of accreditation and how this process supports sustained improvements to the quality of patient care. Employees at the managerial level are involved in the planning, training and education, and implementation of all necessary changes to meet the accreditation standards, and thus a group who can provide these in-depth insights.

The purpose of this study was to explore the perceptions of hospital managers in Saudi Arabian Joint Commission International (JCI) accredited hospitals on the benefits and difficulties of accreditation, and their experiences of the accreditation process. Our overall aim was to better understand JCI accreditation as it is enacted in Saudi hospitals in order to generate recommendations for future accreditation processes.

Our research questions were: what are managers' view about the benefits and difficulties of accreditation in Saudi hospitals and how do managers plan for and experience the accreditation process?

\section{METHODS}

Data were collected between 2 January and 12 February 2016.

\section{Interview questions}

The interview questions were developed on the basis of a review of existing literature to identify accreditation-relevant concepts. Nine questions were developed concerning participants' views on reasons for undertaking accreditation; changes put in place to achieve it; challenges, benefits and disadvantages; 


\begin{tabular}{ll}
\hline Table 1 & Participant codes \\
\hline Code & Location or participant role \\
\hline H1 & Hospital one \\
H2 & Hospital two \\
H3 & Hospital three \\
HD & Hospital Director \\
MD & Medical Director \\
QMD & Quality Management Director \\
ICD & Infection Control Director \\
PHD & Pharmacy Director \\
ND & Nursing Director \\
OTD & Operation Theatre Director \\
PAD & Patient Affairs Director \\
\hline
\end{tabular}

ability to maintain performance after accreditation; and future recommendations.

\section{Sample and data collection}

Three accredited public hospitals were selected from a total of nine accredited hospitals in Saudi Arabia, taking into account diversity in geographical location, bed capacity and scope of service. A purposive sampling technique was employed to recruit participants with expertise across the different JCI accreditation standards such as infection control, leadership and quality. The study was undertaken between one and 3 years post-accreditation in each of the participating hospitals.

The sample population for this research comprised senior managers (hospital directors, medical directors, nurse directors, quality directors and heads of departments) of three accredited hospitals in Saudi Arabia. We provided hospital directors with a participant information sheet (PIS) and asked them to send the PIS to their managers. Those willing to participate contacted the research team. We undertook purposive sampling to ensure diversity in experience and views. Sampling continued until no new ideas were emerging from interviews. ${ }^{1}$

All participants were asked whether they wanted to be interviewed in Arabic or English. Interviews were audiorecorded. They were then transcribed and sent back to participants for review and ratification of the content. Following transcription, interviews undertaken in Arabic were translated into English by an independent translator. Data were de-identified prior to analysis and participants were assigned codes to ensure confidentiality (table 1 ).

\section{Data analysis}

We followed Braun and Clarke's six-step process for systematic thematic analysis. ${ }^{1}$ This involved a thorough review of the transcript for each participant. Statements and meaningful phrases were coded. Codes were compared for similarities and differences then grouped into thematic categories and subcategories. The research team regularly met to discuss emerging themes before themes were decided on.

\section{RESULTS}

Of the 24 invited, 20 managers participated in interviews. Participant characteristics are outlined in table 2.

\section{Thematic findings}

The following five inter-related themes emerged concerning the JCI accreditation process and its impact on quality of patient care: drivers for the change; plan for the change; the process of the change; maintenance of the changes after accreditation; and patient issues (table 3).

\section{Drivers for the change}

Becoming accredited by an international organisation is voluntary in some countries and compulsory in others such as Saudi Arabia and some Gulf countries. Participants in this study indicated that the Ministry of Health was under political and social pressure to accredit

Table 2 Participant characteristics

\begin{tabular}{|c|c|c|c|}
\hline & Hospital site 1 & Hospital site 2 & Hospital site 3 \\
\hline Participants $\mathbf{N}=20$ & $6(30 \%)$ & $7(35 \%)$ & $7(35 \%)$ \\
\hline Nationality & Saudi & Non-Saudi & \\
\hline \multirow[t]{2}{*}{ Gender } & Male & Female & \\
\hline & 17 (85\%) & $3(15 \%)$ & \\
\hline Age & $9(45 \%)$ & $7(35 \%)$ & $4(20 \%)$ \\
\hline \multirow[t]{2}{*}{ Educational level } & Bachelor & Master & Fellowship/PhD \\
\hline & $9(45 \%)$ & $5(25 \%)$ & $6(30 \%)$ \\
\hline
\end{tabular}

The managers interviewed in this research came from different managerial levels - top, middle and front-line managers - and different work areas. These included hospital administration, medical, nursing, pharmacy, operation theatre, quality, infection prevention and control and patient affairs. 


\begin{tabular}{|c|c|}
\hline Theme & Elements \\
\hline $\begin{array}{l}\text { 1. Drivers for } \\
\text { the change }\end{array}$ & $\begin{array}{l}\text { Compulsory from Ministry of Health. } \\
\text { To improve healthcare quality and } \\
\text { patient safety. } \\
\text { Reputational institutional and personal } \\
\text { gains. }\end{array}$ \\
\hline $\begin{array}{l}\text { 2. Plan for the } \\
\text { change }\end{array}$ & $\begin{array}{l}\text { Action plans. } \\
\text { Organisation structure. } \\
\text { Human resources. } \\
\text { Employee culture. }\end{array}$ \\
\hline $\begin{array}{l}\text { 3. The process } \\
\text { of the change }\end{array}$ & $\begin{array}{l}\text { Changes in infrastructure. } \\
\text { Technology system improvement. } \\
\text { Documentation. } \\
\text { Applying high-level standards. } \\
\text { Staff culture. } \\
\text { Training, knowledge and workload. }\end{array}$ \\
\hline $\begin{array}{l}\text { 4. Maintaining } \\
\text { changes post- } \\
\text { accreditation }\end{array}$ & $\begin{array}{l}\text { Follow-up and commitment by } \\
\text { leadership. } \\
\text { Incentives and punishment. } \\
\text { - Manpower. } \\
\text { Clinical quality measures. } \\
\text { Education and training. } \\
\text { - Pressure on hospital. } \\
\text { - Frequent changes in management. } \\
\text { Staff culture. }\end{array}$ \\
\hline $\begin{array}{l}\text { 5. Patients' } \\
\text { issues }\end{array}$ & $\begin{array}{l}\text { Improves patients' care and satisfaction. } \\
\text { Decreased medical errors. } \\
\text { Improves quality and patients' safety. }\end{array}$ \\
\hline
\end{tabular}

hospitals, and this pressure flowed on to them to apply for accreditation. Participants said that despite this push from the Ministry, hospitals genuinely wanted to improve their quality and safety.

The first reason for joining JCI was to achieve patient safety as the JCI focuses on patient safety issues. (PHD, H2)

Accreditation is very important and I am confident that quality means safety. An example of that is the 'time out' [during the surgical safety checklist], which was not implemented before the JCI and it was the first International Patient Safety Goal (IPSG) standard to identify the patient correctly. We had sentinel events in most of the country's hospitals on a daily basis. It happens when a patient is taken to the OT instead of another [OT]. But after implementing 'time out', which involves identifying the patient on the table, the events were eliminated. (HD, H2)

Additionally, participants said they thought international accreditation improved their hospital's reputation among the public. People wanted to be treated at an accredited hospital.

The first benefit we gained from being an accredited hospital is the good reputation as being internationally accredited. (ND, H2)
We became a referral hospital and patients want to be treated in our hospital. Patients' trust in physician is a proof of their satisfaction. (OTD, H3)

This increase in public trust translated to financial gains for the hospitals.

From my point of view, the only reason for joining the JCI accreditation was to increase hospital's budget. It was mainly financial and not for organisational improvement (PHD, H3)

\section{Plan for the change}

The accreditation process involved a lot of planning by managers. Participants said they had to first familiarise themselves with all requirements for the accreditors' visit. Next, they invested heavily in resources to comply with the JCI standards and they made organisational structural changes.

The JCI require safety from $\mathrm{A}$ to $\mathrm{Z}$ such as exit doors, fire extinguishers, and other machines to be connected with an alarm system which was not available before the JCI. (QMD, H2)

A lot of changes have to be made in order to attain accreditation starting with the organisational structure, the leadership design. The leadership responsibility should be clear, clear organisational structure should be in place, design pathways, implementing a system thinking, and leadership commitment towards accreditation. (QM, H3)

Participants said the planning stage for accreditation put significant pressure on both themselves and other hospital staff. The workload was reportedly very high during the accreditation process. This was coupled with workforce shortages and tight timelines to achieve significant changes.

The hardest challenge we faced was the manpower shortage which does not give you enough time to adopt ideas or new work. This required us to work hard and work extra hours to cover this shortage. We worked continuously until 12 midnight until we achieved it. (MD, H2)

While structural changes were difficult to achieve, cultural changes were considered even more difficult. Successful quality improvement initiatives require time, training and resources. Yet participants reported limitations in each of these areas. Participants said they believed If you have qualified staff things will go smoothly (PHD, H1). But getting staff upskilled was difficult within tight time frames, particularly with workforce shortages. So cultural change was slow.

Training must be given in a flexible way and for enough time. For example, heads of departments must be trained for between 3 and 12 months. These trained heads must then be empowered and change those who are non-productive. (QMD, H2) 
In cases where participants had skilled, motivated staff the cultural change towards higher quality and safety was quicker and less stressful.

As there are qualified employees who are aware of the JCI accreditation standards, we will be able to deliver better service and reach the highest quality standards of care. (PHD, H1)

Participants noted that cultural change was key to maintaining the quality and safety changes created through the accreditation process.

If you want to improve quality you must first change society and organisational culture. After that you can implement any quality programme through involving all employees. (ND, H1)

The first thing that needed to be changed was the culture of the organisation itself. The people were not familiar with the accreditation process so we needed a total cultural change in the institution regarding the quality and patient safety. (MD, H3)

One way participants motivated staff to work hard and create cultural change within the context of workforce shortages and tight timelines was with financial incentives.

\section{Process of the change}

The staff resist the change because they are used to routine work and any new work requires time and effort. (MD, H1)

Making large changes in any organisation can be difficult to achieve. The significant challenges identified by our participants were in the following areas: infrastructure, for example, creating new rooms, emergency exits, relocating services such as pharmacy or radiology to align with accreditation standards; new technological resources associated human resources including electronic medical records, automated pharmaceutical dispensing systems; and new hospital processes for delivery safe care such as the WHO Surgical Safety Checklist.

An example of making change that was time-expensive but valued was changed to medical record documentation. With accreditation, all procedures need to be documented and tracked. Participants said the JCI accreditation process had improved their documentation.

There is a big difference in documentation than before accreditation. You document everything you do which is a good thing itself. Documentation protects you and makes those who come after you start from where you ended. (PTD, H1)

Participants valued such changes because they were evidence-based and could lead to better patient care.

The JCI accreditation standard affects the care because it is evidence-based and people implement it in the right, and scientific way, under the supervision of surveyors who are considered experts in their fields. (ICD, H1)
JCI Accreditation standards were designed to measure performance. Participants valued such measures.

In God we believe, others should bring data, no one could improve what he cannot measure. (QM, H1)

Making the required changes to policies, procedures and department plans came at a cost however: participants said the time hospital staff spent completing documentation in patients' files was significantly increased, leaving less time for them to spend with patients.

The biggest disadvantage of accreditation is the more documentation it adds to the work. Paperwork increased notably in the presence of shortage in manpower. (ND, H2)

It actually adds more paperwork which in turn affects the time spent with patients. For example, admitting a patient before accreditation took 10 minutes compared to post-accreditation which takes a much longer time because every single thing has to be documented. (PAD, H3)

In terms of making cultural change, as noted above, this was slow and difficult to achieve. Participants found strategies that promoted the kind of cultural change they wanted including involving all employees in the accreditation process.

Employees have to be involved in the process because leaders alone cannot make all changes in the hospital. (QMD, H3)

Participants found these strategies largely effective but they noted some staff were difficult to engage with and motivate to make changes. This was often due to staff having insufficient knowledge of how to apply the accreditation standards to their own practice and because their workloads were so high that they could not achieve change.

The resistance to change was the hardest obstacle that we faced from employees and heads of departments. They did not want to change even for the best which can lead to patient safety. Employees do not concern themselves about outcomes but daily work. The resistance was due to workloads. (ND, H1)

When specifically asked about strategies they implemented to overcome resistance to change, participants mentioned strategies including training, education and force.

We have a very strong management who sent circulars to all departments and forced people to comply through appointing strict heads. (ICD, H3)

Staff resistance was overcome by education and training and they were involved in the process making because they are directly dealing with these process and standards. (ND, H3) 
The culture of instilling change in people was a bit difficult but we could do it by regular education of people, regular communication at all levels like doctors, nurses and technicians. (MD, H3)

\section{Maintaining changes post-accreditation}

Achieving accreditation was hard work. However, maintaining the level of performance achieved during accreditation was-in some cases-even harder. To maintain changes post-accreditation participants described embedding monitoring strategies with regular staff surveys, committees and the documentation and evaluation of particular processes. These strategies were largely reported by participants in middle management. Top management was less engaged.

The follow-up by the top management decreased after achieving accreditation which caused a drop in performance. A follow-up from the top management is required in order to continue at the same level we achieved during accreditation. (PTA, H2)

Resources were available during accreditation process but discontinued immediately after achieving accreditation, which affected the continuity of care. For example, whatever we requested during accreditation we got immediately but not after achieving accreditation. (ND, H1)

Top management commitment is the most important principle of quality. I read this and did not believe it until I saw it myself and started believing it is the only solution to quality. If you want to implement quality, you need a management who believes in it. (QMD, H1)

Top management should motivate employees financially to maintain quality. (PH, H1).

Management tried different approaches to encourage staff to maintain accreditation changes. Some participants said they used financial deprivation and appointing very strict department heads to motivate staff to maintain changes.

We as a quality management department used the accreditation to threaten employees that they would not be included in the excellence allowance list if we failed accreditation or re-accreditation. (QMD, H2).

From my point of view punishment is the best way that helped us get back to the performance reached during accreditation. (MD, H1)

We have a very strong management who sent circulars to all departments and forced people to comply through appointing strict heads. (ICD, H3)

Other participants used financial rewards to encourage staff to maintain changes.

Employees were rewarded, especially those who worked hard during and after accreditation. Also their names were listed among those eligible for excellence allowances this year which is $10 \%$ of their basic salaries, in recognition of their efforts. (ND, $\mathrm{H} 2$ )

Incentives aside, participants faced the very real problem of maintaining a workforce capable of maintaining the changes. Accreditation put work pressure on hospital staff, and this pressure eventually led some hospital processes to deteriorate.

Maintaining performance after accreditation is harder than achieving it. The reason is the lack of continuity due to healthcare professional shortage. This shortage is due to vacations or resignations for unknown reasons. (MD, $\mathrm{H} 1$ )

We have a problem that the experienced employees are resigning and new ones are coming and we have to teach them again and again so that they will reach the same level. (ND, H3)

I noticed a drop in performance in my department after we achieved the JCI accreditation. Medication errors have increased after accreditation due to not complying with standards. (PHD, H1)

To mitigate this, participants said continuous staff education was key.

We have a quality department that has strategies on how to maintain performance after accreditation. These strategies included training and education of all employees on quality aspects. (PA, H3)

We have to educate employees to keep them all at the same level as their counterparts. There is annual training in quality and patient safety for all employees, refreshers for the existing employees and induction courses for the new ones. (QMD, H3)

Again, the education was sometimes coupled with punitive strategies for those not attending.

Attendance for lectures and training was high because it is mandatory. If you did not attend, you had to give an excuse and they would schedule you in another one. There was also punishment for those not attending. (PAD, H3)

Maintaining change was difficult to achieve in the context of staff turnover occurring at the top and middle management levels.

\section{Patient issues}

Participants described the importance of their accreditation processes in terms of patient safety and quality of care. This final theme explores their prioritisation of patients in their narratives of accreditation. First, participants reported that patients motivated the initial plan for their institution to gain accreditation. Once accreditation was gained participants reported higher patient engagement, more patients accessing their services and improved patient outcomes. 
Medication errors decreased such as dispensing errors, prescribing errors from doctors, and administration errors. We compared the number of errors before and after accreditation and noticed improvement after accreditation. (PHD, H1)

Regarding patient safety, we have indicators of morbidity and mortality, sentinel events, near miss, and medication errors and noticed improvement in all indicators after accreditation. (HD, H2)

Patients reported higher rates of satisfaction and participants genuinely thought this was based on improvements in the quality of care their hospital was providing since gaining accreditation. However, as time progressed, performance sometimes dropped and according to participants, this was due to both increased number of patients needing care and difficulties in maintaining accreditation changes.

There is an increase in the number of patients which is an important reason behind a drop in performance, which I considered positive and negative at the same time. This increase is due to patients' willingness to be treated in our hospital or transferred from other hospitals. On one side it reflects the good reputation the hospital achieved after accreditation, and on the other side it impacted the continuity of care and caused drop in performance. (HD, H2)

\section{DISCUSSION}

The inter-related themes that emerged concerning the JCI accreditation process and its perceived impact on quality of patient care focused around drivers for the change, planning for the change, the process of the change, maintaining changes post-accreditation and patient issues. Participants were positive in their accounts of (1) drivers for the change, (2) planning changes needed to achieve accreditation and (3) managing patient issues. However, participants reported less favourably on the processes of implementing and maintaining changes post-accreditation. ${ }^{8}$

Undertaking accreditation by an international organisation seemed to be driven by different goals. Some positive drivers were compulsion from the Ministry of Health, to improve quality and patient safety, and for enhancing reputation as an accredited hospital. Planning the change was the first step for accreditation by an international organisation. At this stage, participating hospitals had extensive plans for wide ranging changes in structure and processes. These organisational changes are deemed a key priority for organisations who seek to attain accreditation. ${ }^{9}$ The changes included but were not limited to infrastructure, documentation and staff culture. However, implementation of the planned changes was more difficult, and often faced resistance from employees. This resistance was driven by time constraints, employee shortage, workload and stress on employees.
Maintaining performance improvements achieved after accreditation was seen as important but difficult. Reasons proposed by interviewees included lack of follow-up and commitment by top management, staff shortages, lack of ongoing incentives, insufficient staff training and frequent changes in management. A study of accredited public hospitals in the United Arab Emirates ${ }^{10}$ emphasised the importance of hospital leadership in realising accreditation.

Perceived ongoing positive changes from accreditation included expansion in the services provided, empowerment of employees through the implementation process, improved employee culture and improved employees' skills. There was also a perception of ongoing improvements in quality, safety and patient satisfaction. However, participants were unable to provide any quantitative data to support their claims.

Although managers perceived accreditation as a tool that improved some aspects of healthcare quality, they also wondered if the considerable resources expended on the exercise could have been spent more productively on other initiatives.

In our study, the three included hospitals implementing JCI accreditation were confronted by many obstacles and challenges, often made worse by limited time frames and insufficient financial support. One wonders if moving too rapidly towards adopting accreditation, potentially with insufficient staff training and preparation for such external programmes, could be a reason behind employee lack of knowledge and resistance. This view is supported by a systematic review published in 2013, which concluded that there is a lack of evidence to support the idea that accreditation benefits justify the cost spent on it. ${ }^{11}$ Using a Strengths, Weaknesses, Opportunities, Threats analysis to investigate the impact of the accreditation process on quality improvement in hospitals, $\mathrm{Ng}$ et $a l^{12}$ pointed out that resistance to change, lack of knowledge, insufficient training and staff workload are critical obstacles to accreditation implementation.

Participants in our study identified several advantages in accreditation, including patient safety and satisfaction, increases in hospitals' budgets, documentation improvement, less paperwork as these accredited hospitals transformed to electronic records, improved infrastructure, traceability of activities and employee empowerment. This adds to the existing knowledge of manager views from other countries. For $\mathrm{Ng}$ et $a l,{ }^{12}$ the opportunities arising from accreditation involved improved patient safety, enhanced public recognition and supplemented market benefits. de Oliveira and Matsuda ${ }^{13}$ in their interview study of hospital quality managers indicated that hospital accreditation results in enhanced quality of care. Our participants reported that while this was initially their experience too, patient safety and quality of care sometimes dropped after accreditation, which they attributed at least in part to increased patient numbers leading to strain on the system. Overall, our findings support the findings of Melo ${ }^{14}$ who concluded that accreditation contributed to improvement in quality of care and patient 
safety, and contradict that of Alshamsi et $a l^{15}$ who concluded that accreditation increased psychosocial risks resulting from increased work demands during accreditation.

\section{Limitations}

We interviewed staff working in three accredited public hospitals in order to obtain a diverse representation of staff views from accredited Saudi hospitals. The hospitals were of various sizes and different locations. Despite this diversity, the views reported here may be culturally specific and not generalisable to other regions. Bias could arise from the interviewer's previous role as a staff nurse in Saudi hospitals, and personal views of accreditation may have influenced the course of interviews and the interpretation of the data. However, we used different methods to minimise researcher bias including double coding, two researchers agreeing on the themes and checking back with participants.

\section{CONCLUSION}

The findings of this qualitative study revealed that the hospital managers participating in the interviews held varying and conflicting views both in favour of and against accreditation programmes. Although some stated that accreditation is a valid tool for improvement, many cast doubt on its effect and reported that it is a costly exercise. There was criticism over the pressure the process of accreditation put on employees and difficulties in maintaining the level of performance achieved for the accreditation. Given the investment on accreditation, and the lack of consensus on its enduring impact, a more cautious approach to accreditation may be indicated. In particular, more time and thought may be needed in preparing for accreditation, investing in staff training and resourcing, and a commitment to maintain the improvements into the future.

Acknowledgements We would like to thank the participating hospitals for their cooperation during conducting this research.

Contributors A A contributed to the extraction of data, analysis, writing and reviewing of this manuscript. All other authors contributed to writing and reviewing this manuscript.

Funding The authors have not declared a specific grant for this research from any funding agency in the public, commercial or not-for-profit sectors.

Competing interests None declared.

Patient and public involvement Patients and/or the public were not involved in the design, or conduct, or reporting, or dissemination plans of this research.

Patient consent for publication Not applicable.

Ethics approval This study involves human participants and was approved by University of Auckland Human Ethics Committee, Reference 013234. Participants gave informed consent to participate in the study before taking part.
Provenance and peer review Not commissioned; externally peer reviewed.

Data availability statement Data are available upon reasonable request. All data relevant to the study are included in the article or uploaded as supplementary information. Not applicable.

Open access This is an open access article distributed in accordance with the Creative Commons Attribution Non Commercial (CC BY-NC 4.0) license, which permits others to distribute, remix, adapt, build upon this work noncommercially, and license their derivative works on different terms, provided the original work is properly cited, appropriate credit is given, any changes made indicated, and the use is non-commercial. See: http://creativecommons.org/ licenses/by-nc/4.0/.

\section{REFERENCES}

1 Braun V, Clarke V. Using thematic analysis in psychology. Qual Res Psychol 2006;3:77-101.

2 Chen J, Rathore SS, Radford MJ, et al. JCAHO accreditation and quality of care for acute myocardial infarction. Health Aff 2003;22:243-54.

3 Sack C, Scherag A, Lütkes P, et al. Is there an association between hospital accreditation and patient satisfaction with hospital care? A survey of 37,000 patients treated by 73 hospitals. Int J Qual Health Care 2011;23:278-83.

4 El-Jardali F, Jamal D, Dimassi H, et al. The impact of hospital accreditation on quality of care: perception of Lebanese nurses. Int $J$ Qual Health Care 2008;20:363-71.

5 Algahtani H, Aldarmahi A, Manlangit J, et al. Perception of hospital accreditation among health professionals in Saudi Arabia. Ann Saudi Med 2017;37:326-32.

6 Melo S. The impact of accreditation on healthcare quality improvement: a qualitative case study. J Health Organ Manag 2016;30:1242-58.

7 Kousgaard MB, Thorsen T, Due TD. Experiences of accreditation impact in general practice - a qualitative study among general practitioners and their staff. BMC Fam Pract 2019;20:146.

8 Al Mansour A, Merry A, Hamblin R, et al. The effect of joint Commission International (JCl) accreditation on healthcare quality: a study of hospitals in Saudi Arabia. Auckland: The university of Auckland, 2018.

9 Brubakk K, Vist GE, Bukholm G, et al. A systematic review of hospital accreditation: the challenges of measuring complex intervention effects. BMC Health Serv Res 2015;15:1-10.

10 Al Mohammed GA. Perceptions of governance of hospital quality of care and accreditation in the United Arab Emirates: a qualitative study. $J$ health informatics dev ctries 2020;14:1-12.

11 Mumford V, Forde K, Greenfield D, et al. Health services accreditation: what is the evidence that the benefits justify the costs? Int J Qual Health Care 2013;25:606-20.

$12 \mathrm{Ng}$ GKB, Leung GKK, Johnston JM, et al. Factors affecting implementation of accreditation programmes and the impact of the accreditation process on quality improvement in hospitals: a SWOT analysis. Hong Kong Med J 2013;19:434-46.

13 Oliveira JLCde, Matsuda LM. Benefits and difficulties in the implementation of hospital accreditation: the voice of quality managers. Escola Anna Nery 2016;20:63-9.

14 Melo S. The impact of accreditation on healthcare quality improvement: a qualitative case study. $J$ Health Organ Manag 2016;30:1242-58.

15 Alshamsi Al, Thomson L, Santos A. What impact does accreditation have on workplaces? A qualitative study to explore the perceptions of healthcare professionals about the process of accreditation. Front Psychol 2020;11:01614. 